\title{
Severe hypoglycemia as a preventable risk factor for cardiovascular disease in patients with type 2 diabetes mellitus
}

\author{
Soo-Yeon Choi and Seung-Hyun Ko
}

Division of Endocrinology and Metabolism, Department of Internal Medicine, St. Vincent's Hospital, College of Medicine, The Catholic University of Korea, Suwon, Korea

Received: June 29, 2020

Accepted: September 5, 2020

\section{Correspondence to}

Seung-Hyun Ko, M.D.

Division of Endocrinology and Metabolism, Department of Internal Medicine, St. Vincent's Hospital, College of Medicine, The Catholic University of Korea 93 Jungbu-daero, Paldal-gu, Suwon 16247, Korea

Tel: +82-31-249-8155

Fax: $+82-31-253-8898$

E-mail: kosh@catholic.ac.kr https://orcid.org/0000-00033703-1479
Glucose-lowering medication and lifestyle modification are essential for optimal glycemic control in patients with type 2 diabetes mellitus (T2DM). However, glucose-lowering agents, particularly insulin and insulin secretagogues, may cause hypoglycemia, which has multiple negative effects on the cardiovascular (CV) system and may cause death. Previous studies using institutional data from the Korean Nationwide Health Insurance database have consistently found a causal relationship between severe hypoglycemia and CV outcomes and mortality. Screening for high-risk patients, appropriate management, and intensive individualized education are the most effective measures and essential for the prevention of harmful hypoglycemic events. Based on identified risk factors that predict severe hypoglycemia, we developed an 1-year risk prediction model for severe hypoglycemia that can be used in clinical settings. In this review, we describe the current understanding of severe hypoglycemia and the clinical implications in patients with T2DM. Furthermore, we highlight the importance of intensive individualized education for high-risk patients and the risk prediction model to reduce severe hypoglycemia.

Keywords: Diabetes mellitus, type 2; Severe hypoglycemia; Cardiovascular diseases; Education

\section{INTRODUCTION}

The rising prevalence of type 2 diabetes mellitus (T2DM) has increased the global burden of diabetic complications, morbidity, mortality, and healthcare costs. Achieving optimal glycemic control with glucose-lowering agents and lifestyle modification are the only effective means to prevent or minimize diabetes-related complications in patients with $\mathrm{T}_{2} \mathrm{DM}[1]$. However, patients may find it difficult to maintain a healthy lifestyle and adhere to pharmacological treatment over a lifetime. Moreover, T2DM is a chronic metabolic disease with progressive deterioration of the beta-cell reserve, and many patients eventually require insulin treatment [2]. Furthermore, as the proportion of older people with multiple morbidities and polypharmacy increases, intensive glycemic control with insulin in those with long-standing, advanced T2DM (i.e., absolute endogenous insulin deficiency) increases the risk of hypoglycemia [2-4].

Hypoglycemia is a major barrier to achieving optimal glycemic control in patients with T2DM. Treatment-induced hypoglycemia is associated with an increased risk of cardiovascular (CV) events and all-cause mortality in patients with type 1 diabetes mellitus (TiDM) and T2DM [5]. Moreover, hypoglycemia has a negative 
impact on emotional well-being, cognitive function, and quality of life, particularly in patients with severe hypoglycemia (SH), which negates the benefits of strict glycemic control. Hypoglycemic events incur substantial economic and psychosocial costs for patients, their family members, and society [6].

Prevention is recognized as the most effective strategy for the management of hypoglycemia in patients with T2DM. Therefore, an understanding of adverse CV outcomes related to $\mathrm{SH}$ and screening for patients with $\mathrm{T} 2 \mathrm{DM}$ at high risk for $\mathrm{SH}$ are of particular importance.

\section{DEFINITION OF HYPOGLYCEMIA IN DIABETES}

The American Diabetes Association and Endocrine Society (ADA/ES) workgroup on hypoglycemia defined hypoglycemia in diabetes as all episodes of abnormally low plasma glucose concentrations that expose the individual to potential harm [7]. Hypoglycemia may be a frequent outcome of glucose-lowering treatments, particularly insulin and insulin secretagogues. In contrast to the diagnosis of clinical hypoglycemia, which is based on Whipple's triad (symptoms consistent with hypoglycemia, a low plasma glucose concentration, and the resolution of symptoms after the plasma glucose concentration is raised to normal), the presence of relevant symptoms and signs is not a prerequisite for the diagnosis of diabetic hypoglycemia because episodes may be asymptomatic, in which individuals are unaware of low blood glucose levels. The ADA/ES workgroup recommended that a plasma glucose concentration $\leq 70 \mathrm{mg} / \mathrm{dL}$ serve as an alert for the possibility of a hypoglycemic event in patients at risk of hypoglycemia. This value is data driven and approximates the glycemic threshold for activation of physiological glucose counterregulatory systems in nondiabetic individuals. The ADA/ES adapted the three-level iatrogenic hypoglycemia in diabetes clinical classification proposed by the International Hypoglycemia Study Group (Table 1) [8]. Although the overall incidence of hypoglycemia is higher in patients with $\mathrm{T} 1 \mathrm{DM}$ than in those with $\mathrm{T}_{2} \mathrm{DM}$, given that the prevalence of T2DM is approximately 20 -fold greater than that of TiDM and many patients with long-standing $\mathrm{T}_{2} \mathrm{DM}$ ultimately require insulin treatment, most episodes of iatrogenic hypoglycemia occur in patients
Table 1. Three-level classification of iatrogenic hypoglycemia in diabetes [8]

\begin{tabular}{ll}
\hline & \multicolumn{1}{c}{ Glycemic criteria/description } \\
\hline Level 1 & $54 \mathrm{mg} / \mathrm{dL} \leq$ glucose $<70 \mathrm{mg} / \mathrm{dL}$ \\
Level 2 & $\begin{array}{c}\text { Glucose }<54 \mathrm{mg} / \mathrm{dL}, \text { sufficiently low to indicate } \\
\text { serious clinically important hypoglycemia }\end{array}$ \\
Level 3 & $\begin{array}{c}\text { A severe event characterized by altered mental } \\
\text { and/or physical functioning that requires } \\
\text { assistance from another person for recovery }\end{array}$ \\
\hline
\end{tabular}

with T2DM [9-12].

Because the symptoms and signs of hypoglycemia can be nonspecific, patients may not be aware of a hypoglycemic event, or mild episodes that are self-treated may not be remembered or reported. In contrast, although $\mathrm{SH}$ represents a small fraction of the total hypoglycemic burden, estimates of the frequency of $\mathrm{SH}$ are more reliable because they are dramatic events that are difficult to ignore and are more likely to be reported.

\section{CLINICAL IMPLICATIONS OF SEVERE HYPOGLYCEMIA}

SH not only affects quality of life and healthcare costs it also directly contributes to mortality Although profound neuroglycopenia can cause fatal brain injury, the condition is rare; thus, more attention has been focused on the causal association between hypoglycemia and cardiac dysfunction. $\mathrm{SH}$ is a strong predictor of major CV events and mortality in patients with T2DM [13-16], although it is unclear whether this is due to a direct pathophysiological association or whether $\mathrm{SH}$ acts as a marker of vulnerability to CV events. Accumulating observational evidence indicates that several CV events occur during $\mathrm{SH}$ either as a result of hypoglycemia itself or via activation of the sympathoadrenal response. Several underlying pathophysiological processes have been proposed including (1) hemodynamic changes that increase cardiac workload and attenuate myocardial perfusion; (2) arrhythmogenic electrophysiological changes; (3) the induction of a prothrombotic state; and (4) the release of inflammatory cytokines [17]. Ultimately, it is likely that multiple factors contribute to CV events, which can be triggered by $\mathrm{SH}$ in high-risk patients. 


\section{CARDIOVASCULAR EFFECTS OF SEVERE HYPOGLYCEMIA}

Evidence suggests that SH can cause myocardial ischemia. The autonomic nervous system is activated as part of the counterregulatory response to a hypoglycemic episode. Sympathoadrenal activation, which stimulates plasma catecholamine secretion, has profound CV effects including decreased parasympathetic activity. A hypoglycemic clamp study (plasma glucose $54 \mathrm{mg} / \mathrm{dL}$ ) in nondiabetic subjects found that a surge in plasma epinephrine was associated with increased heart rate and stroke volume and decreased peripheral vascular resistance, resulting in an increase in cardiac output and a fall in central arterial pressure [18-20]. Such hemodynamic responses to $\mathrm{SH}$ may be detrimental to patients with long-duration diabetes and underlying heart disease $[21,22]$.

Of further clinical significance is the finding that hypoglycemia can induce cardiac arrhythmias, which may cause sudden death [23-25]. The arrhythmogenic effect of hypoglycemia may act through several mechanisms: a low blood glucose concentration has a direct effect on ion channels, and catecholamines induce hypokalemia and action potential/QT prolongation, which increases the risk of ventricular arrhythmias and sudden death [26-28]. A previous study of lethal hypoglycemia in rats revealed disturbances of cardiac electrical stability. SH initially induced sinus tachycardia, QT prolongation, and atrial fibrillation (AF) followed by ventricular tachycardia, atrioventricular blockade, and eventual bradycardia and cardiorespiratory arrest [29].

The prothrombotic and proinflammatory effects of hypoglycemia may be associated with CV events. Hormones, including catecholamines, released during hypoglycemia increase blood viscosity and promote platelet aggregation and activation [22,30-32]. Furthermore, insulin-induced hypoglycemia has been shown to increase coagulation factors and promote the release of inflammatory cytokines, which may remain elevated for several days after hypoglycemia is corrected [31-35]. These proinflammatory and procoagulant effects together with endothelial dysfunction can directly affect vascular flow for some time after a hypoglycemic episode and may contribute to the development of CV events.
Despite the suggestion of a causal relationship between SH and CV events reported by mechanistic studies, evidence from large prospective studies is limited. A causal relationship between $\mathrm{SH}$ and CV events is supported by the findings of the Action in Diabetes and Vascular Disease: Preterax and Diamicron-MR Controlled Evaluation (ADVANCE), the Action to Control Cardiovascular Risk in Diabetes (ACCORD), and the Outcome Reduction With Initial Glargine Intervention (ORIGIN) studies, all of which found higher rates of hypoglycemia-related mortality in the intensive treatment arm than in the standard treatment arm $[15,16,36]$. However, the association between $\mathrm{SH}$ and $\mathrm{CV}$ events is strongest in patients at high CV risk Currently available evidence suggests that $\mathrm{SH}$ can trigger $\mathrm{CV}$ events in vulnerable patients with high CV risk; therefore, hypoglycemia should be avoided in patients with known cardiovascular disease (CVD) [37].

\section{INSTITUTIONAL RESEARCH FINDINGS: ASSO- CIATION BETWEEN SEVERE HYPOGLYCEMIA AND CARDIOVASCULAR EVENTS}

\section{Hospital-based cohort study}

To investigate the association between $\mathrm{SH}$ and the risk of CVD or all-cause mortality in patients with T2DM, we analyzed the data of 1,260 patients with T2DM recorded in the St. Vincent's Hospital registry. During the 10.4year follow-up period, $9.4 \%$ of the patients experienced at least one $\mathrm{SH}$ episode. SH was significantly associated with an increased risk of all-cause mortality (hazard ratio [HR], 2.64; 95\% confidence interval [CI], 1.39 to 5.02; $p=0.003$ ) and CVD mortality (HR, 6.34; 95\% CI, 2.02 to 19.87; $p=0.002$ ) after adjusting for sex, age, diabetes duration, mean glycosylated hemoglobin level, diabetic nephropathy, hypertension, lipid profile, and insulin use [38].

$\mathrm{SH}$ is associated with cardiac arrhythmia; therefore, we investigated the change in heart-rate-corrected QT interval (QTc) during SH. We reviewed medical records including the electrocardiograms of 208 patients with T2DM who visited the emergency department with SH. The QTc was measured during SH and 6 months before and after SH. During SH, 54.8\% of the patients had QTc prolongation followed by a significant decrease in QTc 
within 6 months of SH (QTc prolongation after recovery, 33.8\%; $p<0.001$ ). The prolonged QTc was significantly associated with baseline QTc prolongation (odds ratio, 2.92; 95\% CI, 1.22 to $6.96 ; p=0.016$ ) after adjusting for multiple confounders [39].

\section{Korean National Health Insurance Service database}

The Korean National Health Insurance Service (NHIS) program established a computerized database (DB) containing all claims data, including patient demographics, drug prescriptions, diagnostic codes based on the disease coding system, the International Classification of Diseases, insurers' payment coverage, patients' deductibles, and claimed treatment details. Therefore, because the Korean NHIS DB is representative of the Korean population, the data are useful for a population-based nationwide study of patients with T2DM [40]. Six diabetes-related CV complications, including ischemic heart disease, acute myocardial infarction, ischemic stroke, hemorrhagic stroke, percutaneous coronary intervention, and coronary artery bypass graft were identified in the NHIS claims DB.

To investigate the association between $\mathrm{SH}$ and new-onset AF and all-cause mortality in adult patients with $\mathrm{T} 2 \mathrm{DM}$, retrospective data were extracted from the NHIS DB. Of 1,509,280 patients with T2DM who received healthcare checkups between 2005 and 2008, $10,864(0.72 \%)$ had experienced $\mathrm{SH}$ in the 3 years before the checkup. During 8.5 years of follow-up, 3.24\% of those patients developed new-onset $\mathrm{AF}$ (3.5 per 1,000 person-years). The incidence of AF was significantly higher in patients who had experienced $\mathrm{SH}$ than in those who had not experienced $\mathrm{SH}$ (7.09 and 3.79 per 1,000 person-years, respectively; $p<0.001$ ). After multivariable adjustment, previous $\mathrm{SH}$ was identified as a significant risk factor for the development of AF (HR, 1.10; 95\% CI, 1.01 to 1.19). After adjustment for multiple variables, all-cause mortality was significantly higher in patients who experienced previous $\mathrm{SH}$ events (HR, 1.57; 95\% CI, 1.50 to 1.64) and in those with prior SH and subsequent $\mathrm{AF}$ onset (HR, 4.64; 95\% CI, 4.55 to 4.74 ) than in patients who had not experienced $\mathrm{SH}$ [41].

We further investigated the associations between $\mathrm{SH}$ episodes and CVD (newly developed myocardial infarction, stroke, heart failure) and all-cause mortality in patients with T2DM. The incidence of adverse outcomes tended to be higher in the group who experienced $\mathrm{SH}$. Experiencing three or more $\mathrm{SH}$ episodes was associated with an approximately 2-fold higher risk of each type of CV event and a 3.28-fold higher risk of all-cause mortality. After adjusting for confounding factors, the HR of myocardial infarction significantly and sequentially increased: o vs. 1 episode (HR, 1.56; 95\% CI, 1.46 to 1.64); O vs. 2 episodes (HR, 1.86; 95\% CI, 1.61 to 2.15); and o vs. 3 or more episodes (HR, 1.86; 95\% CI, 1.48 to 2.35; $p$ for trend < 0.001). Similarly, the number of $\mathrm{SH}$ episodes was associated with sequential increases in the risk of stroke, heart failure, and all-cause mortality. Moreover, we found a dose-response relationship between the number of SH events and all main outcomes ( $p$ for trend $<$ o.001). The risks for all CV outcomes and mortality were highest within 1 year of the index date and showed decreasing trends on follow-up. Significant results from the dose-response, temporal, and sensitivity analyses suggest direct causality between SH and CV outcomes and mortality [42].

\section{PREVENTION OF SEVERE HYPOGLYCEMIA}

\section{Screening of high-risk patients: a risk prediction model for $\mathrm{SH}$}

Given the negative effects of $\mathrm{SH}$ on diabetes management and CV outcomes, the ability to predict $\mathrm{SH}$ accurately and manage the risk factors before an $\mathrm{SH}$ episode is essential for patients with T2DM. For this reason, we developed a 14-variable prediction model to estimate the 1-year risk of $\mathrm{SH}$ in patients with $\mathrm{T} 2 \mathrm{DM}$ using the NHIS DB. Age, sex, smoking status, alcohol drinking status, body mass index, exercise, previous SH episode, use of insulin or multiple oral hypoglycemic agents, the presence of hypertension or chronic kidney disease, duration of diabetes, glucose level, and the Charlson Comorbidity Index score were included in the model(Figs. 1 and 2) [43].

Using this risk model, we created an interactive webbased calculator for the 1-year risk of SH (http://md.koobian.com/sh/index.html) for practical use in the clinical setting (Fig. 2B). Our model showed good calibration and discrimination with a C-statistic index of 0.871 ( $95 \%$ CI, 0.863 to 0.881 ) [43]. This risk scoring system will enable healthcare professionals to screen patients at high 

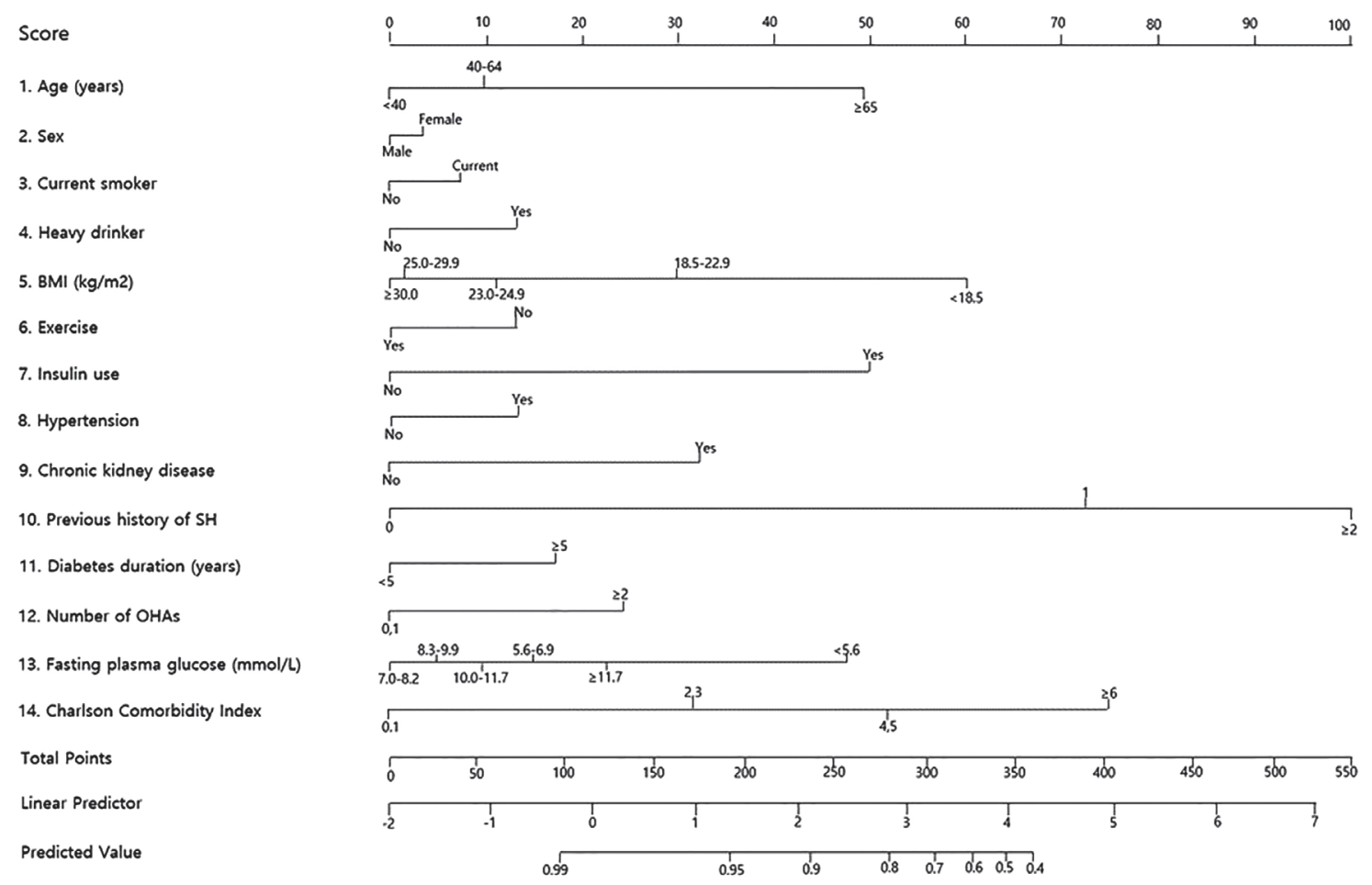

Figure 1. Predictive nomogram for the 1-year probability of severe hypoglycemia (SH). Adapted from Han et al. [43]. BMI, body mass index; OHA, oral hypoglycemic agent.

risk for $\mathrm{SH}$ easily, and the score can be immediately incorporated into medical decision making such as adjusting the dose of the hypoglycemic agent, determining the cause of hypoglycemia, and providing intensive patient education. Therefore, our prediction model could reduce the incidence of $\mathrm{SH}$ in high-risk $\mathrm{T} 2 \mathrm{DM}$ patients.

\section{Diabetes self-management education: the role and effectiveness of intensive individualized reinforce- ment education for the prevention of hypoglycemia in patients with $\mathrm{T}_{2} \mathrm{DM}$}

Patient education plays an essential role in diabetes care, particularly for the prevention of SH. Well-organized, intensive patient education delivered by certified professional healthcare providers has been shown to be cost-effective and to improve glycemic control, self-care behavior, well-being, and reduce CV risk factors [4446]. Previously, we reported that a structured, intensive group education program helped patients with $\mathrm{T}_{2} \mathrm{DM}$ improve their dietary habits, engage in more frequent physical activity and regular self-monitoring of their glucose levels, and achieve better glycemic control [47].
Furthermore, intensive individualized education can help patients with T2DM at high risk of SH manage hypoglycemia and avoid SH episodes [48]. Important skills taught in the education program include the adjustment of insulin or insulin secretagogues dose and recognizing the early signs of hypoglycemia. Detection of low glucose concentration with no symptoms is critically important because it is indicative of impaired awareness of hypoglycemia, a treatable condition of conventionally defined severe iatrogenic hypoglycemia caused by an even lower glucose concentration [49]. Therefore, individualized intensive education with reinforcement and regular monitoring will reduce the number of hypoglycemic episodes and ultimately reduce related major CV events and mortality in patients at high risk for $\mathrm{SH}$ [50].

\section{CONCLUSIONS}

Healthcare professionals who treat patients with $\mathrm{T}_{2} \mathrm{DM}$ in the clinical setting should be aware of the potential detrimental effects of hypoglycemia on prognosis, ma- 


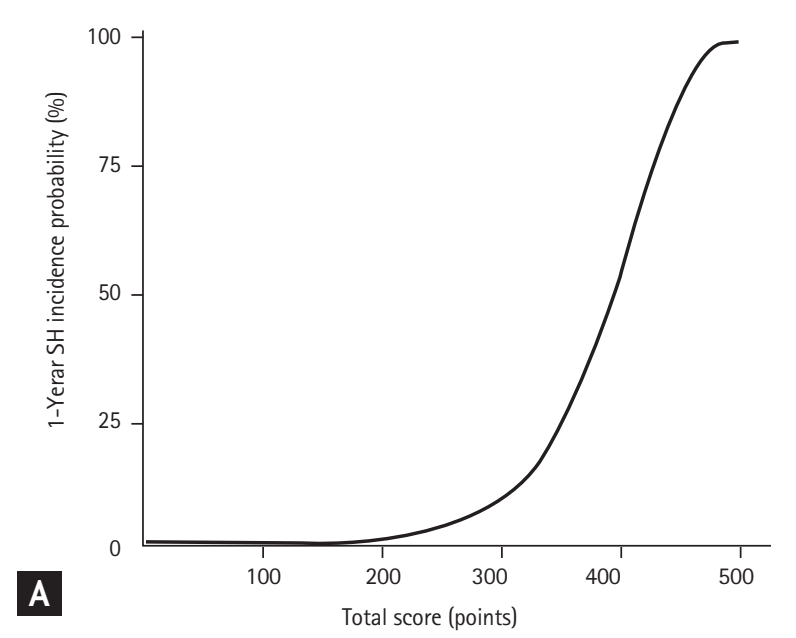

Result

Total points : 290

Figure 2. (A) The 1-year incidence probability of severe hypoglycemia (SH) according to the total score. (B) An example of risk prediction for $\mathrm{SH}$ in a 66-year-old woman with type 2 diabetes mellitus (no smoking or alcohol habits, body mass index 23.8 $\mathrm{kg} / \mathrm{m}^{2}$, moderate intensity exercise, on insulin treatment, hypertension, chronic kidney disease, previous history of an severe hypoglycemic event, diabetes duration of 10 years, fasting glucose $122 \mathrm{mg} / \mathrm{dL}$, one class of oral hypoglycemic agent, and Charlson Comorbidity Index score of 2 points) using the risk prediction model (http://md.koobian.com/sh/index.html). Adapted from Han et al. [43].

jor CV events, and mortality. Based on accurate assessment of SH risk, immediate intervention should be considered for patients deemed high risk, including setting a less stringent glycated hemoglobin target, modifying antidiabetic management, increasing self-monitoring of plasma glucose levels, adopting lifestyle modification practices and regular mealtimes, and $\mathrm{SH}$ education. Of these interventions, we believe that intensive individualized education is the most fundamental and effective way to prevent SH. Therefore, it is reasonable to devote considerable attention and medical resources to education for patients with T2DM at high risk of SH. Moreover, with technological advances, the availability of continuous glucose monitoring systems (CGMS) has increased. Wider use of CGMS will improve understanding of blood glucose patterns and glucose management; moreover, CGMS provide a more precise measurement of blood glucose levels than self-monitoring. Therefore, accurate CGMS data will allow the development of more individualized and tailored $\mathrm{SH}$ prevention education programs.

\section{Conflict of interest}

No potential conflict of interest relevant to this article was reported.

\section{REFERENCES}

1. Kim MK, Ko SH, Kim BY, et al. 2019 Clinical practice guidelines for type 2 diabetes mellitus in Korea. Diabetes Metab J 2019;43:398-406.

2. Hepburn DA, MacLeod KM, Pell AC, Scougal IJ, Frier BM. Frequency and symptoms of hypoglycaemia experienced by patients with type 2 diabetes treated with insulin. Diabet Med 1993;10:231-237.

3. UK Hypoglycaemia Study Group. Risk of hypoglycaemia in types 1 and 2 diabetes: effects of treatment modalities and their duration. Diabetologia 2007;50:1140-1147.

4. United Kingdom Prospective Diabetes Study Group. United Kingdom Prospective Diabetes Study 24: a 6-year, randomized, controlled trial comparing sulfonylurea, insulin, and metformin therapy in patients with newly diagnosed type 2 diabetes that could not be controlled with diet therapy. Ann Intern Med 1998;128:165-175.

5. Khunti K, Davies M, Majeed A, Thorsted BL, Wolden ML, Paul SK. Hypoglycemia and risk of cardiovascular disease and all-cause mortality in insulin-treated people with type 1 and type 2 diabetes: a cohort study. Diabetes Care 2015;38:316-322.

6. Kim G, Lee YH, Han MH, et al. Economic burden of hypoglycemia in patients with type 2 diabetes mellitus from Korea. PLoS One 2016;11:e0151282. 
7. Seaquist ER, Anderson J, Childs B, et al. Hypoglycemia and diabetes: a report of a workgroup of the American Diabetes Association and the Endocrine Society. Diabetes Care 2013;36:1384-1395.

8. International Hypoglycaemia Study Group. Glucose concentrations of less than $3.0 \mathrm{mmol} / \mathrm{l}$ (54 mg/dl) should be reported in clinical trials: a joint position statement of the American Diabetes Association and the European Association for the Study of Diabetes. Diabetes Care 2017;40:155-157.

9. Cho NH, Kim NK, Han E, et al. Patient understanding of hypoglycemia in tertiary referral centers. Diabetes Metab J 2018;42:43-52.

10. Adler AI, Stratton IM, Neil HA, et al. Association of systolic blood pressure with macrovascular and microvascular complications of type 2 diabetes (UKPDS 36 ): prospective observational study. BMJ 2000;321:412-419.

11. ADVANCE Collaborative Group, Patel A, MacMahon S, et al. Intensive blood glucose control and vascular outcomes in patients with type 2 diabetes. $\mathrm{N}$ Engl J Med 2008;358:2560-2572.

12. Ismail-Beigi F, Craven T, Banerji MA, et al. Effect of intensive treatment of hyperglycaemia on microvascular outcomes in type 2 diabetes: an analysis of the ACCORD randomised trial. Lancet 2010;376:419-430.

13. Bedenis R, Price AH, Robertson CM, et al. Association between severe hypoglycemia, adverse macrovascular events, and inflammation in the Edinburgh type 2 diabetes study. Diabetes Care 2014;37:3301-3308.

14. McCoy RG, Van Houten HK, Ziegenfuss JY, Shah ND, Wermers RA, Smith SA. Increased mortality of patients with diabetes reporting severe hypoglycemia. Diabetes Care 2012;35:1897-1901.

15. ORIGIN Trial Investigators, Mellbin LG, Ryden L, et al. Does hypoglycaemia increase the risk of cardiovascular events? A report from the ORIGIN trial. Eur Heart J 2013;34:3137-3144.

16. Zoungas S, Patel A, Chalmers J, et al. Severe hypoglycemia and risks of vascular events and death. N Engl J Med 2010;363:1410-1418.

17. International Hypoglycaemia Study Group. Hypoglycaemia, cardiovascular disease, and mortality in diabetes: epidemiology, pathogenesis, and management. Lancet Diabetes Endocrinol 2019;7:385-396.

18. Laitinen T, Huopio H, Vauhkonen I, et al. Effects of euglycaemic and hypoglycaemic hyperinsulinaemia on sympa- thetic and parasympathetic regulation of haemodynamics in healthy subjects. Clin Sci (Lond) 2003;105:315-322.

19. Fisher BM, Gillen G, Hepburn DA, Dargie HJ, Frier BM. Cardiac responses to acute insulin-induced hypoglycemia in humans. Am J Physiol 1990;258(6 Pt 2):H1775-H1779.

20. Hilsted J, Bonde-Petersen F, Norgaard MB, et al. Haemodynamic changes in insulin-induced hypoglycaemia in normal man. Diabetologia 1984;26:328-332.

21. Hanefeld M, Duetting E, Bramlage P. Cardiac implications of hypoglycaemia in patients with diabetes: a systematic review. Cardiovasc Diabetol 2013;12:135.

22. Wright RJ, Frier BM. Vascular disease and diabetes: is hypoglycaemia an aggravating factor? Diabetes Metab Res Rev 2008;24:353-363.

23. Leitch A. Sudden death in insulin coma treatment for schizophrenia. J Nerv Ment Dis 1955;121:267-269.

24. Tanenberg RJ, Newton CA, Drake AJ. Confirmation of hypoglycemia in the "dead-in-bed" syndrome, as captured by a retrospective continuous glucose monitoring system. Endocr Pract 2010;16:244-248.

25. Tattersall RB, Gill GV. Unexplained deaths of type 1 diabetic patients. Diabet Med 1991;8:49-58.

26. Al-Khatib SM, LaPointe NM, Kramer JM, Califf RM. What clinicians should know about the QT interval. JAMA 2003;289:2120-2127.

27. Nordin C. The proarrhythmic effect of hypoglycemia: evidence for increased risk from ischemia and bradycardia. Acta Diabetol 2014;51:5-14.

28. Zhang $Y$, Han $H$, Wang J, Wang $H$, Yang B, Wang Z. Impairment of human ether-à-go-go-related gene (HERG) $\mathrm{K}+$ channel function by hypoglycemia and hyperglycemia. Similar phenotypes but different mechanisms. J Biol Chem 2003;278:10417-10426.

29. Reno CM, Daphna-Iken D, Chen YS, VanderWeele J, Jethi K, Fisher SJ. Severe hypoglycemia-induced lethal cardiac arrhythmias are mediated by sympathoadrenal activation. Diabetes 2013;62:3570-3581.

30. Frier BM. Hypoglycaemia in diabetes mellitus: epidemiology and clinical implications. Nat Rev Endocrinol 2014;10:711-722.

31. Hutton RA, Mikhailidis D, Dormandy KM, Ginsburg J. Platelet aggregation studies during transient hypoglycaemia: a potential method for evaluating platelet function. J Clin Pathol 1979;32:434-438.

32. Wright RJ, Newby DE, Stirling D, Ludlam CA, Macdonald IA, Frier BM. Effects of acute insulin-induced hypoglyce- 
mia on indices of inflammation: putative mechanism for aggravating vascular disease in diabetes. Diabetes Care 2010;33:1591-1597.

33. Dalsgaard-Nielsen J, Madsbad S, Hilsted J. Changes in platelet function, blood coagulation and fibrinolysis during insulin-induced hypoglycaemia in juvenile diabetics and normal subjects. Thromb Haemost 1982;47:254-258.

34. Dandona P, Chaudhuri A, Dhindsa S. Proinflammatory and prothrombotic effects of hypoglycemia. Diabetes Care 2010;33:1686-1687.

35. Gogitidze Joy N, Hedrington MS, Briscoe VJ, Tate DB, Ertl AC, Davis SN. Effects of acute hypoglycemia on inflammatory and pro-atherothrombotic biomarkers in individuals with type 1 diabetes and healthy individuals. Diabetes Care 2010;33:1529-1535.

36. Bonds DE, Miller ME, Bergenstal RM, et al. The association between symptomatic, severe hypoglycaemia and mortality in type 2 diabetes: retrospective epidemiological analysis of the ACCORD study. BMJ 2010;340:b4909.

37. Pistrosch F, Ganz X, Bornstein SR, Birkenfeld AL, Henkel E, Hanefeld M. Risk of and risk factors for hypoglycemia and associated arrhythmias in patients with type 2 diabetes and cardiovascular disease: a cohort study under real-world conditions. Acta Diabetol 2015;52:889-895.

38. Cha SA, Yun JS, Lim TS, et al. Severe hypoglycemia and cardiovascular or all-cause mortality in patients with type 2 diabetes. Diabetes Metab J 2016;40:202-210.

39. Cha SA, Yun JS, Lim TS, et al. Baseline-corrected QT (QTc) interval is associated with prolongation of QTc during severe hypoglycemia in patients with type 2 diabetes mellitus. Diabetes Metab J 2016;40:463-472.

40. Ko SH, Han K, Lee YH, et al. Past and current status of adult type 2 diabetes mellitus management in Korea: a National Health Insurance Service database analysis. Diabetes Metab J 2018;42:93-100.

41. Ko SH, Park YM, Yun JS, et al. Severe hypoglycemia is a risk factor for atrial fibrillation in type 2 diabetes mellitus: Nationwide population-based cohort study. J Diabetes Complications 2018;32:157-163.

42. Yun JS, Park YM, Han K, Cha SA, Ahn YB, Ko SH. Severe hypoglycemia and the risk of cardiovascular disease and mortality in type 2 diabetes: a nationwide population-based cohort study. Cardiovasc Diabetol 2019;18:103.

43. Han K, Yun JS, Park YM, et al. Development and validation of a risk prediction model for severe hypoglycemia in adult patients with type 2 diabetes: a nationwide population-based cohort study. Clin Epidemiol 2018;10:15451559 .

44. Norris SL, Lau J, Smith SJ, Schmid CH, Engelgau MM. Self-management education for adults with type 2 diabetes: a meta-analysis of the effect on glycemic control. Diabetes Care 2002;25:1159-1171.

45. Rhee EJ, Kim HC, Kim JH, et al. 2018 Guidelines for the management of dyslipidemia. Korean J Intern Med 2019;34:723-771.

46. Kim Y, Ahn Y, Cho MC, Kim CJ, Kim YJ, Jeong MH. Current status of acute myocardial infarction in Korea. Korean J Intern Med 2019;34:1-10.

47. Ko SH, Song KH, Kim SR, et al. Long-term effects of a structured intensive diabetes education programme (SIDEP) in patients with type 2 diabetes mellitus: a 4-year follow-up study. Diabet Med 2007;24:55-62.

48. Yun JS, Ko SH. Avoiding or coping with severe hypoglycemia in patients with type 2 diabetes. Korean J Intern Med 2015;30:6-16.

49. Yong YM, Shin KM, Lee KM, et al. Intensive individualized reinforcement education is important for the prevention of hypoglycemia in patients with type 2 diabetes. Diabetes Metab J 2015;39:154-163.

50. de Zoysa N, Rogers H, Stadler M, et al. A psychoeducational program to restore hypoglycemia awareness: the DAFNE-HART pilot study. Diabetes Care 2014;37:863-866. 